\section{Author reply: A further caveat in interpreting cancer survival}

Isabelle Soerjomataram and Esther de Vries

We would like to thank Drs Rosso and Zanetti for their useful addition to the topics discussed in our Perspective. ${ }^{1}$ We agree on the growing importance of multiple cancers and the need to improve documentation on both studies of multiple cancers and common coding rules between countries, to avoid differences as described by Rosso and Zanetti.

In our Perspective we mentioned the role of comorbidity (including multiple cancers) in the determination of survival and changes in survival over time, although the role of multiple cancers could have been more explicitly included in the discussion. ${ }^{1}$ Rosso and Zanetti argue that including multiple-cancer cases in analyses will often lead to lower relative survival estimates compared to survival estimates of patients with only one cancer diagnosed. Yet, it is not clear whether inclusion of data for multiple cancers would lead to a worsening in survival estimates over time. If increasing numbers of multiple cancer survivors (due to aging and improved treatment) indeed causes a decline in survival estimates, we would expect to see continuously decreasing survival estimates in the future, as the prevalence of multiple cancers is deemed to increase.

We are of the opinion, however, that improvements in cancer care and early detection have also benefited those with multiple cancers. In addition, patients with a previous cancer diagnosis tend to be more aware of cancer risks and generally receive more check-ups. ${ }^{2}$ Therefore, the stage distribution of a second cancer is generally better than that of first cancers ${ }^{3}$ which probably leads to good treatment outcome. In addition, lead time and possibly length bias might have a role in improved survival among these patients (this is explained in more detail in our Perspective). ${ }^{1}$ Furthermore, studies that have examined survival of patients with multiple cancers did not always show significantly worse outcomes among those with a second cancer. ${ }^{2}$ This observation was also reported for metachronous breast cancer diagnosed more than 10 years after the first cancer; survival was not worse compared with women with a single breast cancer. ${ }^{4}$ Finally, even among the oldest cancer registries, continuous improvement in survival over time has been observed despite aging and increasing numbers of patients with multiple cancers. ${ }^{5}$

Currently, there is not enough evidence to suggest that an increase in the number of patients with multiple cancers has caused a worsening of cancer survival estimates over time. Nevertheless, the contribution of multiple cancers to survival estimates is gaining in importance and therefore should be taken into consideration in the estimation of cancer burden.

Department of Public Health, Erasmus MC University Medical Center Rotterdam, PO Box 2040, 3000 CA Rotterdam, The Netherlands (I. Soerjomataram, E. de Vries).

Correspondence to: E. de Vries e.devries@erasmusmc.nl

doi:10.1038/nrclinonc.2009.184-c2

Competing interests

The authors declare no competing interests.

1. de Vries, E. et al. Explanations for worsening cancer survival. Nat. Rev. Clin. Oncol. 7, 60-63 (2010).

2. Sankila, R. \& Hakulinen, T. Survival of patients with colorectal carcinoma: effect of prior breast cancer. J. Natl Cancer Inst. 90, 63-65 (1998).

3. Soerjomataram, I., Louwman, W. J., Duijm. L. E. \& Coebergh, J. W. Rising incidence of breast cancer among female cancer survivors: implications for surveillance. Br. J. Cancer 100, 77-81 (2009).

4. Hartman, M. et al. Incidence and prognosis of synchronous and metachronous bilateral breast cancer. J. Clin. Oncol. 25, 4210-4216 (2007).

5. Karim-Kos, H. E. et al. Recent trends of cancer in Europe: a combined approach of incidence, survival and mortality for 17 cancer sites since the 1990s. Eur. J. Cancer 44, 1345-1389 (2008). 